\title{
Presencia actual de la fonética en el aula de ELE y su reflejo en la enseñanza de la prosodia
}

\author{
Gabino Boquete Martín \\ Universidad de Alcalá \\ gabino@alcalingua.com
}

Resumen: Los estudios de fonética en el aula de ELE tradicionalmente han carecido del tratamiento que se merecen. La aplicación de los actuales modelos comunicativos en la enseñanza de lenguas debería haber ayudado a mejorar su presencia y utilización como elemento fundamental para conseguir una buena competencia comunicativa. El propósito de este artículo es comprobar cuál es la situación de la enseñanza de la fonética (concretamente la prosodia) en nuestros días; para ello se realiza un trabajo de investigación con estudiantes y profesores de español, en la Universidad de Alcalá, sobre las preferencias y uso de las diferentes competencias comunicativas, así como las actitudes y creencias de los participantes hacia el estudio la fonética. Así mismo, se examina la tipología de los ejercicios de práctica prosódica y el tratamiento que se da a la fonética en los manuales de ELE disponibles en el mercado. Esta investigación confirma el poco interés que la enseñanza y aprendizaje de la fonética sigue despertando (insignificante en el caso de la prosodia en español), la necesidad de preparación teórica complementaria por parte del profesor y cómo el contacto de los estudiantes con esta disciplina es casi nulo. Esta falta de atención se ve reflejada en los materiales didácticos, en la metodología y en la práctica diaria en el aula de lengua.

Palabras claves: fonética, prosodia, manuales de ELE, competencia comunicativa, tipología de ejercicios. 


\begin{abstract}
Traditionally, phonetic studies at Spanish as a Foreign Language classroom have lacked the treatment they deserve. The application of current communicative models in language teaching should help to improve their presence and practice as a key to achieving good communication skills. The purpose of this article is to verify the status of Phonetics teaching (specifically the Spanish Prosody) nowadays; for this reason we have prepared a research with students and teachers of Spanish, at the University of Alcalá, focused on their preferences and use of different communication skills, together with the attitudes and beliefs of the participants towards the study of Phonetics. This work also examines the typology of prosodic exercises and the treatment given to the manuals available in the market. This research corroborates the lack of interest concerning the teaching and learning of Phonetics (negligible in the case of Prosody), the need for additional theoretical preparation by the teachers and how the students contact with this discipline is almost nonexistent. This neglect is reflected in teaching materials, methodology and daily practice in the language classroom.
\end{abstract}

Key words: Phonetics, prosody, Spanish as Second Language manuals, communicative skills, exercises typology. 


\section{Introducción}

El uso de las destrezas orales en el aula de lengua, gracias a la incorporación de los métodos comunicativos a su enseñanza, ha ido adquiriendo una importancia fundamental en los últimos años (Boquete, 2014). A pesar de ello, según Cantero (2002), Cortés (2002), Carbó (2003), Gil (2007), Gil (2012) e Hidalgo y Cabedo (2012) entre otros investigadores, el trato que se le da al estudio de la pronunciación y corrección fonética en general es poco más que testimonial. Se evita tratar contenidos fonéticos y de pronunciación en el aula, sobre todo aquellos que tienen que ver con los patrones rítmicos y entonativos característicos de la lengua meta, como algo realmente importante en el proceso de adquisición.

El problema reside en la propia complejidad del estudio de la fonética y en que los profesores de lenguas no cuentan, por lo general, con la preparación necesaria para enseñar las técnicas de pronunciación y corrección fonética (Gil, 2007). El profesor de lenguas no va a tener que explicar toda la información relacionada con esta disciplina; Cortés (2002:61) pone como ejemplo: «el alumno no necesita saber los parámetros que subyacen a una sílaba acentuada — tono, intensidad, duración y timbre - ni para percibirla ni para producirla», pero debe conocer cuáles son sus fundamentos y necesita poseer las herramientas adecuadas para comunicarlo.

Este desconocimiento y falta de interés se ve reflejado en los materiales de español disponibles en el mercado, en los que el estudio de la fonética tiene una presencia minoritaria (Carbó, 2003); así mismo, existe una mayor investigación y desarrollo de materiales didácticos enfocados al análisis de los elementos segmentales (fonemas) que al trabajo de los elementos suprasegmentales (entonación, ritmo, acento y pausa) (Cantero, 2002). Es necesario incidir más en estos últimos, dada su importancia como elemento indispensable para cohesionar el discurso.

El propósito de este artículo es verificar la vigencia de los estudios mencionados (casi todos realizados hace una década) y comprobar la situación actual de la enseñanza de la fonética en el aula de ELE. Para ello, se lleva a cabo una investigación que arranca en el análisis de las actitudes y creencias de los implicados (profesores y estudiantes de español pertenecientes a la Universidad de Alcalá) sobre las diferentes competencias comunicativas para, a continuación, abordar el estudio en el área de la fonética, la experiencia de los entrevistados respecto a su uso y su presencia en el aula. La investigación centra su foco, a parir de este momento, en la experiencia concreta de los encuestados respecto al estudio de la prosodia en español, su práctica y el tipo de actividades realizadas para su adqui- 
sición. Finalmente, se lleva a cabo un análisis sobre el tratamiento general que los manuales de ELE realizan sobre la fonética.

El objetivo fundamental es ratificar si, tal y como apunta Cortés (2002), la enseñanza de la prosodia sigue siendo una asignatura pendiente en la enseñanza de lenguas. No es posible alcanzar una buena competencia comunicativa sin un dominio suficiente de la prosodia, ya que un error en la entonación, por ejemplo, puede producir equivocaciones más graves y malentendidos con repercusiones más negativas en el proceso comunicativo que un uso gramatical inadecuado (Sosa, 1999; Canellada y Kuhlmann, 1987; Cortés, 2002). Por ello, con este artículo queremos defender que es esencial prestar mucha más atención al estudio de los elementos suprasegmentales en el currículo de la enseñanza de lenguas.

Será fundamental, para la actualización y contraste de datos, el análisis de las encuestas realizadas a profesores de español y a estudiantes internacionales. El ámbito de estudio lo compone, en cuanto a profesores, 40 participantes en diferentes situaciones profesionales y experiencia variada; el número de estudiantes entrevistados, universitarios, con una edad media de 23 años, supera los 200 y engloba a estudiantes de Estados Unidos, Asia y Europa con una participación muy equilibrada de cada entorno cultural. También se llevará a cabo un análisis de la tipología de ejercicios que se utiliza en el aula en la actualidad, así como el tratamiento de la fonética en los manuales de ELE disponibles.

\section{La enseñanza de los elementos prosódicos y su tratamiento en el aula}

Cuando se estudia una lengua extranjera no se tiene que prestar atención sólo al léxico y a la gramática sino también a la pronunciación y la corrección fonética, porque estos aspectos forman un todo. Por lo tanto, el estudiante que desee aprender bien una lengua extranjera debe adquirir también una nueva base de articulación, debe acostumbrarse a articular los sonidos extranjeros e intentar distanciarse de los hábitos propios de su lengua materna (Gil, 2012).

Los términos fonética y pronunciación se entremezclan a menudo y confunden al estudiante de lenguas (también a los profesionales, como se puede percibir en la denominación que se usa en los manuales de ELE o en las propias programaciones que realizan los profesores). Carbó (2003) establece las diferencias: la pronunciación está orientada a la producción correcta de los elementos segmentales y suprasegmentales de la lengua, es una especialidad dentro de las destrezas orales para la que el profesor debe tener un conocimiento amplio de la fonética. La pronunciación y la corrección fonética se enmarcan en la enseñanza de la expresión oral; la enseñanza de la fonética sirve de ayuda en este cometido. 
La mayoría de los autores coincide en las peculiaridades de la entonación como componente fundamental de la prosodia. Sin ir más lejos, Quilis (1999: 409) deja claro que: «El suprasegmento entonativo es uno de los componentes más complejos de una lengua: sus variadas formas, sus diversas funciones, su incidencia más o menos directa en la significación y en la caracterización del hablante lo convierten en un campo de estudio en el que no faltan las dificultades».

Los asuntos relativos a la gramática y al léxico son más fáciles de analizar; la capacidad de introspección por parte de los hablantes y de los estudiosos es más clara hacia los aspectos que se refieren al significado global que hacia la melodía. Los fenómenos melódicos están condicionados por la expresión oral, siempre más individual y subjetiva. En este sentido, en su estudio sobre la entonación, Sosa (1999: 19) afirma: «Los hechos entonativos, contrariamente a lo que sucede con otros aspectos de la sustancia fónica y con los demás niveles de análisis lingüístico, son difíciles de describir con precisión perceptualmente, y difíciles también de caracterizar en términos de componentes, de segmentos, o de rasgos, dentro de un modelo coherente».

Por lo tanto, sería conveniente, antes de nada, precisar el ámbito de estudio en el que se va a desarrollar la investigación que se acometerá en la parte empírica de este artículo: Cortés (2002) define la prosodia como la rama de la Lingüística que analiza y representa formalmente aquellos elementos no verbales de la expresión oral. Se asocia a las variaciones de la frecuencia fundamental, duración e intensidad y a otros elementos derivados de la vibración de las cuerdas vocales como la pausa, el acento y el ritmo. Por consiguiente, en este artículo, los términos prosodia y elemento suprasegmental harán mención a una misma realidad.

La prosodia yace en la estructura profunda del lenguaje; no es un fenómeno que simplemente acompañe al lenguaje o que añada a los enunciados determinados matices gramaticales y pragmáticos. Por el contrario, es un fenómeno esencial del lenguaje humano y, por tanto, de la competencia comunicativa. Canellada y Kuhlman (1987: 75) mantienen que no hablamos con vocablos o con palabras aisladas, sino que estos van encajados en una línea tonal general, y que esta línea tonal es fundamental para la comprensión del mensaje: «la entonación es fundamental en el estudio o el conocimiento de un idioma. Según el tono, un sonido, una palabra, un simple gruñido a veces, puede expresar la duda, la pregunta, el enfado, la repulsa».

Cada ser humano tiene un modo personal de hablar que es parte de su identidad como individuo y que va ligada a la de una colectividad, la de su propia lengua materna. Este modo de hablar se ve reflejado cuando se estudia una lengua extranjera, en el que los hablantes muestran, en mayor o menor medida, rasgos fó- 
nicos característicos de su lengua materna, aun cuando sean capaces de alcanzar un nivel sobresaliente en otros planos de la lengua extranjera (léxico, gramatical, estilístico o pragmático). Cortés (1999) afirma que la pronunciación es el componente esencial de la identidad lingüística, y que, dentro de la pronunciación, la entonación es el subcomponente más 'íntimo' para el hablante, ya que revela una información sumamente personal: actitud, estado de ánimo, sentimientos y emociones.

En general se asume que la entonación puede ser considerada uno de los aspectos más difíciles de asimilar y de enseñar. Cortés (2002) así lo hace constar y expone la falta de atención que generalmente ha tenido la entonación en la enseñanza de segundas lenguas. Una de las causas apuntadas por el autor es que los estudios fonológicos se han centrado en los elementos segmentales, los fonemas, dejando de lado la acentuación, el ritmo y la entonación. Una de las misiones del docente es hacer ver al estudiante que es tan importante pronunciar de una forma adecuada y aplicar una entonación acorde con el discurso como construir frases gramaticalmente correctas (Hidalgo y Cabedo, 2012).

Pero la realidad es que los profesores de lenguas no cuentan, por lo general, con la preparación necesaria para acometer la labor de enseñar las técnicas de pronunciación y entonación, tanto en el caso de lengua materna como en el de segundas lenguas. Además, en el improbable caso de contar con ese conocimiento, debe ser transmitido de una forma sencilla y clara al estudiante, con lo que la misión se vuelve más compleja. Gil (2007) confirma esta idea e invita a los fonetistas a que aporten nuevos métodos didácticos que le sean útiles al docente en su tarea.

En este cometido el profesor cuenta con la ayuda del Plan Curricular del Instituto Cervantes (PCIC) en su sección dedicada al estudio de los elementos suprasegmentales $^{1}$ («pronunciación y prosodia», según su propia terminología) en la clase de ELE. El Instituto Cervantes (2006) parte de la siguiente reflexión: la forma en que un extranjero pronuncia la lengua del país que lo acoge influye en la consideración de la comunidad hacia él; generalmente será mejor aceptado cuanto más se acerque al modelo nativo, consiguiendo una mayor aceptación social e incluso mayores perspectivas de desarrollo profesional y de relaciones personales. Por tanto, la aspiración de la mayoría de los estudiantes de lenguas extranjeras debería ser la de acercarse a la pronunciación de un nativo. Se incide en la necesidad de realizar una orientación «ecléctica y de naturaleza integradora», en la que confluyan las aportaciones positivas de cada modelo existente:

Todo el debate existente en el campo de la enseñanza de lenguas acerca del grado de mayor o menor atención que se le debe conceder a la forma (frente al con-

1 Los contenidos completos pueden consultarse en Boquete (2012) anexo II 2.5.4.2. 
tenido o la función) tiene una cierta razón de ser si nos referimos al componente estrictamente gramatical de la lengua (morfología, sintaxis...), pero deja de tenerla si estamos hablando de pronunciación, porque en este campo la atención a la forma es simplemente imprescindible.

(Instituto Cervantes, 2006: 128)

No se trata, en palabras del PCIC, de caer en el énfasis exagerado del aspecto formal, que provoque dificultades a la hora de emitir enunciados reales o responder a ellos de una forma natural. El profesor debe estar preparado académicamente para adaptarse sobre la marcha, y ser capaz de aplicar el método más adecuado a las necesidades específicas detectadas en los alumnos para, de este modo, integrarlas en el conjunto de actividades del modo más coherente posible. Su papel no puede ser desempeñado ni por un programa informático ni por una grabación, ni ningún aparato, a pesar de que dichas opciones puedan ser utilizadas como un buen complemento en la clase.

\section{Marco metodológico de la investigación}

Con el fin de contrastar las ideas apuntadas en el marco teórico previamente expuesto, y acreditar si estas siguen vigentes en nuestros días, se diseñó un trabajo de investigación cualitativa centrada en preguntas realizadas a profesores $y$ a estudiantes internacionales de español para conocer su percepción sobre el uso del estudio de la fonética en el aula. El estudio se realizó con muestras tomadas en 2012 en un ámbito de investigación muy variado, tanto por la diversidad de nacionalidades como por la variedad de niveles de dominio del español y de años de estudio, en el caso de los estudiantes, o de años de docencia en el caso de los profesores. Esta investigación se llevó a cabo en el centro de enseñanza de español de la Universidad de Alcalá, Alcalingua, y es parte de una tesis doctoral (Boquete, 2012).

Para la confección de las preguntas se utilizó el modelo de 'competencia comunicativa' propuesto por Alcón (2000), que incluye tres subcompetencias relacionadas entre sí por los siguientes rangos: competencia discursiva (competencia lingüística: gramática, fonética y léxico; competencia textual; competencia pragmática), competencias y habilidades psicomotoras (escuchar, hablar, leer y escribir: destrezas lingüísticas) y competencia estratégica (estrategias de comunicación y de aprendizaje). Las preguntas se centraran en la competencia discursiva y las competencias psicomotoras.

En cuanto al análisis de las respuestas, se utilizó el 'modelo de Likert'; en el caso del análisis de la tipología de las actividades de práctica prosódica se manejó 
un tipo de variable cualitativa (dicotómica). El análisis de los datos recabados en las encuestas, basados en 'tablas de frecuencias', se realizó utilizando el paquete estadístico SAS versión 9.1.

\section{A. Ámbito de investigación 'profesores':}

Se inició el estudio centrando el foco en los docentes y su percepción sobre la importancia de la oralidad en el aula a profesores que trabajaban en este centro y a profesores que recibían cursos de formación y de actualización metodológica.

El número de participantes es de 40 profesores en ejercicio y con una experiencia media de 7 años: la gran mayoría (un 70\%) son nativos en lengua española; aún así, se cuenta con profesores de orígenes variados: 2 brasileños, 4 estadounidenses, 1 jordano y 1 tailandés.

B. Ámbito de investigación 'estudiantes':

213 participantes, con una edad media de 22 años; el espacio geográfico y cultural se distribuye del siguiente modo: China 70 (32,9\%); Estados Unidos 27 (12,7\%); Europa 68 (31,9\%); Japón 31 (14,6\%); Otros 17 (8,0\%).

Criterio de distribución: se divide en bloques teniendo en cuenta la proximidad geográfica y cultural de los países, así como por la similitud de sus estilos educativos. El bloque denominado 'Europa' engloba a países como Suiza (1), Ucrania (2) y Rusia (3), además de los pertenecientes a la Unión Europea; la gran mayoría son estudiantes Erasmus.

Otros: Arabia Saudí 2, Australia 1, Brasil 2, Corea del Sur 3, Marruecos 2, Tailandia 1, Taiwán 6. La variable 'otros' tiene muy poca incidencia en el estudio, por lo que su aparición es meramente anecdótica y solo pretende mostrar la variedad de nacionalidades que aparecen.

El nivel de dominio de español, según el Marco Común Europeo de Referencia (MCER), es el siguiente:

A1: 16 (7,7\%); A2: 43 (20,6\%); B1: 71 (34,0\%); B2: 49 (23,4\%); C1: 30 $(14,4 \%)$.

C. Ámbito de investigación 'manuales de ELE'

Por último, y con el fin de analizar la importancia que le dan al estudio de la fonética los manuales de ELE, se procedió a revisar de una muestra de 31 manuales disponibles en el mercado. 


\section{Situación actual del uso de la fonética en el aula de ELEः la prosodia}

A continuación, se procede a analizar los datos obtenidos en las encuestas diseñadas para mostrar cuál es la realidad actual en cuanto a la presencia de los estudios de fonética en el aula de español como lengua extranjera.

Comenzamos por la percepción de los profesores, sus actitudes y creencias en cuanto al reparto de cada una de las competencias comunicativas, del tiempo dedicado a la enseñanza de la fonética en el aula, su apreciación en cuanto a la preparación que un profesor debe tener para transmitir el conocimiento relacionado con la fonética y su prioridad en cuanto a los diferentes elementos fonéticos (segmentos y suprasegmentos).

Acto seguido se da paso a la visión de los estudiantes internacionales de español que asisten a cursos de inmersión lingüística en la Universidad de Alcalá: sus preferencias en cuanto a la oralidad y al uso de la competencia gramatical, de su percepción sobre la formación recibida, en su país de origen, en cuanto a la pronunciación y corrección fonética, su trabajo sobre la prosodia española, etcétera.

Se concluye con la revisión de la tipología de las actividades de práctica prosódica y el análisis de los manuales de ELE en cuanto a su dedicación a la fonética española.

\subsection{Actitudes y creencias de los profesores ante el uso de la fonética en el aula}

\subsubsection{Dedicación ideal del profesor en cuanto a las competencias comunicativas}

La primera pregunta realizada a los entrevistados tiene la intención de establecer las preferencias de los profesores a la hora de repartir la dedicación de cada competencia en el aula. La pretensión de esta pregunta es que los profesores indiquen un reparto ideal según la importancia asignada.

La pregunta planteada es:

Te parece más importante que tus alumnos, ordena de mayor (1) a menor (5) importancia:

( ) Empleen adecuadamente las destrezas orales

( ) Tengan buena pronunciación/corrección fonética

() Tengan mucho vocabulario

() Empleen adecuadamente las destrezas escritas

( ) Usen la gramática correctamente 
El resultado de las prioridades del profesor queda claramente definido en la tabla que se muestra a continuación:

\begin{tabular}{|c|c|c|c|c|c|}
\hline Competencia & $\begin{array}{c}\text { Lo más } \\
\text { importante }\end{array}$ & Importante & Indiferente & $\begin{array}{c}\text { Poco } \\
\text { importante }\end{array}$ & $\begin{array}{c}\text { Lo menos } \\
\text { importante }\end{array}$ \\
\hline Destrezas orales & $32(82,1)$ & $5(12,8)$ & $0(0,0)$ & $0(0,0)$ & $2(5,1)$ \\
\hline Fonética & $2(5,1)$ & $10(25,6)$ & $7(18,0)$ & $9(23,1)$ & $11(28,2)$ \\
\hline Vocabulario & $1(2,6)$ & $4(10,3)$ & $10(25,6)$ & $11(28,2)$ & $13(33,3)$ \\
\hline Destrezas escritas & $1(2,6)$ & $11(28,2)$ & $9(23,1)$ & $12(30,8)$ & $6(15,4)$ \\
\hline Gramática & $3(7,7)$ & $9(23,1)$ & $13(33,3)$ & $7(18,0)$ & $7(18,0)$ \\
\hline
\end{tabular}

Tabla 1: dedicación ideal del profesor - n (\%)

Llama la atención el dominio claro de las destrezas orales (un 82,1\% de los profesores las han valorado como la opción más importante) sobre el resto de destrezas y sobre la gramática. Del mismo modo, solo un 5,1\% ha marcado estas destrezas como lo menos importante. En todo caso, no produce indiferencia, pues ningún entrevistado ha marcado la opción 'indiferente' o 'poco importante.' En Boquete (2014) se desarrolla de forma más detallada la situación actual de las destrezas orales en el contexto de las competencias comunicativas.

En cuanto a la fonética y pronunciación, solo un 5,1\% muestra prioridad por su enseñanza en el aula de ELE, y un número muy alto (el $28,2 \%$ ) piensa que es lo menos importante. Como se puede comprobar, solo el 5,1\% de los entrevistados da prioridad absoluta y un $28,2 \%$ le concede la importancia menor.

\subsubsection{Dedicación real del profesor en cuanto a las competencias comunicativas}

A continuación se contrasta esta imagen ideal con la dedicación real que lleva a cabo el profesor a cada una de las competencias. Por ello, se plantea la siguiente pregunta:

A qué aspectos das prioridad, ordena de mayor (1) a menor (5) dedicación de tiempo:

( ) Comprensión/expresión oral

( ) Pronunciación/corrección fonética

( ) Léxico

( ) Comprensión/expresión escrita

( ) Gramática 
Estos son los resultados obtenidos:

\begin{tabular}{|c|c|c|c|c|c|}
\hline Competencia & $\begin{array}{c}\text { Lo más } \\
\text { importante }\end{array}$ & Importante & Indiferente & $\begin{array}{c}\text { Poco } \\
\text { importante }\end{array}$ & $\begin{array}{c}\text { Lo menos } \\
\text { importante }\end{array}$ \\
\hline Destrezas orales & $30(77,0)$ & $6(15,4)$ & $1(2,6)$ & $0(0,0)$ & $2(5,1)$ \\
\hline Fonética & $2(5,1)$ & $7(18,0)$ & $8(20,5)$ & $9(23,1)$ & $13(33,3)$ \\
\hline Vocabulario & $2(5,1)$ & $4(10,3)$ & $12(30,8)$ & $11(28,2)$ & $10(25,6)$ \\
\hline Destrezas escritas & $2(5,1)$ & $11(28,2)$ & $9(23,1)$ & $9(23,1)$ & $8(20,5)$ \\
\hline Gramática & $3(7,7)$ & $11(28,2)$ & $9(23,1)$ & $10(25,6)$ & $6(15,4)$ \\
\hline
\end{tabular}

Tabla 2: dedicación real del profesor - n (\%)

Sorprende la coincidencia que se da entre el reparto ideal mostrado por el profesor en la tabla anterior (Tabla 1) con el que aplica en la realidad de las clases o de las prácticas. En este caso sigue ganando con mucho la dedicación a las destrezas orales $(77,0 \%)$ sobre el resto de las competencias.

La fonética pierde peso en la dedicación real del profesor y aumenta el porcentaje de entrevistados que consideran su enseñanza como lo menos importante $(33,3 \%$ frente al $28,2 \%$ que aparecía en la Tabla 1$)$. Esto no debe sorprender si se tiene en cuenta que las herramientas con las que cuenta el profesor son más bien escasas. La escasez de bibliografía y de materiales especializados provoca una falta de formación que hace que el profesor se sienta inseguro y preocupado por la posibilidad de enfrentarse a un tema para el que no está preparado y del que carece de información. Por consiguiente, el profesor considera la práctica y enseñanza de la pronunciación como una tarea ardua y difícil de tratar en su clase.

\subsubsection{Conocimientos del profesor con respecto a la fonética}

Para confirmar la sensación de inseguridad del profesor en cuanto a la preparación fonética que necesita, preguntamos:

¿Qué conocimientos de fonética debe tener el profesor para mejorar la pronunciación de sus estudiantes?

Los resultados de esta pregunta son:

Ninguno: 1 (2.6\%); básicos: 2 (5.1\%); amplios: 31 (79.5\%); especializados: $5(12.8 \%)$.

Se puede ver cómo los profesores consideran que para enseñar fonética y mejorar la pronunciación de los estudiantes se debe tener, como mínimo, unos conocimientos amplios. Muchos exigirían conocimientos especializados para impartir el área de fonética a estudiantes de ELE. 


\subsubsection{Los estudios fonológicos se centran en los elementos segmentales}

En el caso de que el profesor trabajara con la fonética, habría que sondear cuáles son los elementos que más le gusta enseñar; por ello, se plantea:

¿Qué aspectos fonéticos preferirías trabajar?: segmentales/suprasegmentales

Los profesores contestan:

Elementos segmentales 24 (64,7\%); elementos suprasegmentales 13 (35,1\%).

Domina, de un modo claro, el estudio de los sonidos sobre el del ritmo, la pausa el acento y la entonación. Algunos entrevistados puntualizan que este aspecto depende del nivel, uno de ellos manifiesta que depende del nivel y de la nacionalidad y otro asigna el estudio de los elementos segmentales a los niveles A1/A2 y el de los suprasegmentales a B1/B2.

\subsection{Actitudes y creencias de los estudiantes en cuanto al estudio de la fonética}

Una vez analizada la importancia que otorgan los profesores a la enseñanza de la fonética, se da paso al estudio de este asunto desde la perspectiva del estudiante. Para ello se realiza un procedimiento similar al de los profesores, esto es, ver la importancia asignada para mostrar un reparto ideal del uso de las competencias $y$, a continuación, contrastarlo con el que realmente han tenido en su país de origen durante su proceso de aprendizaje de español.

\subsubsection{Dedicación ideal del estudiante en cuanto a las competencias comunicativas}

En la primera pregunta dirigida a los estudiantes internacionales se refleja la prioridad que estos dan a cada una de las competencias planteadas:

A la hora de estudiar una lengua lo más importante para mí es, ordena de mayor (1) a menor (5) importancia:

( ) Utilizar bien las destrezas orales (comprender/hablar bien)

( ) Tener buena pronunciación/fonética

() Tener mucho vocabulario

( ) Utilizar bien las destrezas escritas (leer/escribir bien)

( ) Usar la gramática correctamente 
Estos son los resultados obtenidos:

\begin{tabular}{|c|c|c|c|c|c|}
\hline Competencia & $\begin{array}{c}\text { Lo más } \\
\text { importante }\end{array}$ & Importante & Indiferente & $\begin{array}{c}\text { Poco } \\
\text { importante }\end{array}$ & $\begin{array}{c}\text { Lo menos } \\
\text { importante }\end{array}$ \\
\hline Destrezas orales & $121(65,8)$ & $25(13,6)$ & $16(8,7)$ & $12(6,5)$ & $10(5,4)$ \\
\hline Fonética & $12(6,5)$ & $33(18,0)$ & $51(27,7)$ & $37(20,1)$ & $51(27,7)$ \\
\hline Vocabulario & $23(12,5)$ & $56(30,4)$ & $44(23,9)$ & $44(23,9)$ & $17(9,2)$ \\
\hline Destrezas escritas & $4(2,2)$ & $41(22,3)$ & $33(17,9)$ & $43(23,4)$ & $63(34,2)$ \\
\hline Gramática & $23(12,5)$ & $29(15,8)$ & $40(21,7)$ & $49(26,6)$ & $43(23,4)$ \\
\hline
\end{tabular}

Tabla 3: dedicación ideal del alumno - n (\%)

Se percibe cómo, para los estudiantes, las destrezas orales gozan de una preferencia absoluta (con un 65,8\%) y aparece como lo menos importante en solo un $5,4 \%$. En eso coinciden con lo expresado por los profesores en las Tablas 1 y 2. No se detectan grandes diferencias si se estratifican los resultados por la variable 'nacionalidad', lo que indica que es una opinión general.

En cuanto a la pronunciación y corrección fonética, los resultados son claros: solo lo consideran importante un $6,5 \%$, y es percibido como lo menos importante por el $27,7 \%$ de los encuestados.

\subsubsection{Dedicación real del estudiante en cuanto a las competencias comunicativas}

En la Tabla 3 se ha mostrado la dedicación que el estudiante desearía que se le diera a las diferentes competencias según sus intereses y necesidades. A continuación se analiza la dedicación real que han tenido en sus estudios de español antes de llegar a España; indica la importancia efectiva que le han dado sus profesores a cada una de las competencias para ver si esto se corresponde con las expectativas de los estudiantes.

En las clases de español que has recibido antes de llegar a la Universidad de Alcalá, qué aspectos has estudiado más; ordena de mayor (1) a menor (5) dedicación de tiempo:

( ) Comprensión/expresión oral (comprender/hablar)

( ) Pronunciación/corrección fonética

( ) Léxico, vocabulario

( ) Comprensión/expresión escrita (leer/escribir)

( ) Gramática 
Los resultados son los que siguen:

\begin{tabular}{|c|c|c|c|c|c|}
\hline Competencia & $\begin{array}{c}\text { Lo más } \\
\text { importante }\end{array}$ & Importante & Indiferente & $\begin{array}{c}\text { Poco } \\
\text { importante }\end{array}$ & $\begin{array}{c}\text { Lo menos } \\
\text { importante }\end{array}$ \\
\hline Destrezas orales & $31(17,5)$ & $17(9,6)$ & $38(21,5)$ & $51(28,8)$ & $40(22,6)$ \\
\hline Fonética & $13(7,3)$ & $17(9,6)$ & $26(14,7)$ & $39(22,0)$ & $82(46,3)$ \\
\hline Vocabulario & $21(11,9)$ & $52(29,4)$ & $54(30,5)$ & $35(19,8)$ & $15(8,5)$ \\
\hline Destrezas escritas & $22(12,4)$ & $51(28,8)$ & $51(28,8)$ & $31(17,5)$ & $22(12,4)$ \\
\hline Gramática & $90(50,8)$ & $44(22,6)$ & $9(5,1)$ & $20(11,3)$ & $18(10,2)$ \\
\hline
\end{tabular}

Tabla 4: dedicación real del alumno - n (\%)

Llama la atención, a primera vista, el viraje que da esta tabla con respecto a la $n^{\circ}$ 3; las diferencias (lo cual refleja la divergencia que se da entre profesores y estudiantes en el lugar de origen) son espectaculares.

Para comenzar, la gramática acapara la atención absoluta de la dedicación en el aula: el 50,9\% de estudiantes afirma que ha sido lo más importante y el 22,6\% que lo considera importante, desbancando a las destrezas orales que bajan hasta el 17,5\% en su calificación de muy importante y tan solo un 9,6\% que asigna el valor 'importante'. Además, en detrimento de las destrezas orales, un 22,6\% afirma que la dedicación en su país de origen ha sido lo menos importante.

Cae todavía más la percepción sobre la importancia de la corrección fonética (su dedicación en el aula) y aumenta la asignación menos importante, pasando de un 27,7\% marcado en la Tabla 3 a un 46,3\%.

Se ve claramente un desacuerdo entre las preferencias de los estudiantes y lo que los profesores consideran prioritario en el aula de español (quizá en el aula de lengua extranjera en general) en los países de origen de los estudiantes.

\subsubsection{Formación recibida por los estudiantes en cuanto a la fonética}

Los estudiantes perciben que el estudio de la fonética es menos importante que el de otras asignaturas, tal y como se pudo comprobar con el análisis de las Tablas 3 y 4. Además, según ellos, el tiempo dedicado al estudio de la fonética y la pronunciación ha sido 'poco', visto las respuestas ante la siguiente pregunta:

Antes de llegar a la Universidad de Alcalá, ‘cuánto tiempo, si lo comparas con otras de asignaturas (gramática, léxico, escritura, conversación, etc.), le has dedicado al estudio de la fonética y pronunciación del español? 
La respuesta de los estudiantes fue: nada: 22 (11,8\%); poco: 95 (51,1\%); suficiente: 39 (21\%); bastante 24 (12,9\%); mucho: $6(3,2 \%)$.

Es fácilmente reconocible que la opción 'poco' $(51,1 \%)$ domina sobre el resto. Además de esta percepción de que ha dedicado poco tiempo al estudio de la fonética, el estudiante tiene claro que su pronunciación debe ser mejorada. Ante la premisa:

Necesito mejorar mi pronunciación en español

Los estudiantes manifiestan estar totalmente en desacuerdo: 3 (1,4\%); en desacuerdo: 17 (8,0\%); ni de acuerdo/ni en desacuerdo: 32 (15,1\%); de acuerdo 70 (33,0\%); totalmente de acuerdo 90 (42,6\%).

\subsubsection{Importancia de los estudios de prosodia en los estudiantes}

Centrándonos en el reparto de los elementos fonéticos, se puede confirmar que, de aquellos estudiantes que han recibido en algún momento instrucciones sobre fonética o pronunciación, la mayoría han tratado con elementos segmentales:

Si has estudiado fonética y pronunciación española, ¿qué parte has estudiado más? Ordena de mayor (1) a menor (5) importancia:

( ) Las vocales y las consonantes, sus características y forma de pronunciación

( ) Las pausas, las comas, los puntos...

( ) Los acentos: palabras esdrújulas, llanas y agudas

( ) Las sílabas, diptongos, triptongos e hiatos

( ) La entonación

\begin{tabular}{|l|c|c|c|c|c|}
\hline \multicolumn{1}{|c|}{ Contenidos } & $\begin{array}{c}\text { Lo más } \\
\text { importante }\end{array}$ & Importante & Indiferente & $\begin{array}{c}\text { Poco } \\
\text { importante }\end{array}$ & $\begin{array}{c}\text { Lo menos } \\
\text { importante }\end{array}$ \\
\hline $\begin{array}{l}\text { a) Vocales } \\
\text { y consonantes }\end{array}$ & $67(44,7)$ & $35(23,3)$ & $19(12,7)$ & $17(11,3)$ & $12(8,0)$ \\
\hline $\begin{array}{l}\text { b) Pausas, comas, } \\
\text { puntos }\end{array}$ & $3(2,0)$ & $11(7,3)$ & $25(16,7)$ & $42(28,0)$ & $69(46,0)$ \\
\hline c) Acentos & $47(31,3)$ & $46(30,7)$ & $28(18,7)$ & $17(11,3)$ & $11(7,3)$ \\
\hline d) Sílabas & $12(8,0)$ & $36(24,0)$ & $41(27,3)$ & $35(23,3)$ & $26(17,3)$ \\
\hline e) Entonación & $20(13,3)$ & $22(14,7)$ & $37(24,7)$ & $39(26,0)$ & $32(21,3)$ \\
\hline
\end{tabular}

Tabla 5: si has estudiado fonética ¿qué has estudiado más? - n (\%)

De los 213 entrevistados, 63 no han contestado, lo que indica que un $29.6 \%$ no ha estudiado nunca (o no tienen la percepción de haberlo hecho) la pronunciación o corrección fonética. De los 150 restantes, la dedicación a los elementos 
segmentales domina sobre los suprasegmentales; aún así se nota que el estudio de la acentuación en español ocupa un plano importante de la programación de los estudiantes en su país de origen.

\subsubsection{Tipología de las actividades de práctica prosódica}

El poco interés existente por los asuntos relacionados con la prosodia se refleja en la falta de creatividad dominante en el diseño de material didáctico, en el que siguen empleándose sobre todo ejercicios de 'escucha y repite', o bien los clásicos ejercicios de sistematización (drills). Cortés (2001) enumera algunas actividades encaminadas a la adquisición de la prosodia en lenguas extranjeras; nosotros hemos preguntado a los estudiantes sobre el uso de las actividades más comunes, actualmente, en el aula de ELE.

Si has estudiado fonética y pronunciación en español, ¿qué tipo de ejercicios has realizado?

\begin{tabular}{|l|l|}
\hline TIPO DE EJERCICIO & $\%$ SI \\
\hline Ejercicios de 'escuchar y repetir' & 86,0 \\
\hline Grabar tu voz y revisarla con el profesor & 39,8 \\
\hline Ejercicios realizados por ordenador & 46,8 \\
\hline Lecturas en voz alta, imitación del profesor... & 74,0 \\
\hline Acentuación, listas de sílabas acentuadas & 66,5 \\
\hline Marcar las pausas, formar grupos de palabras & 52,5 \\
\hline Distinguir frases interrogativas, exclamativas, afirmativas... & 66,2 \\
\hline Acompañar con movimientos la entonación de una frase & 46,7 \\
\hline Transcripciones fonéticas, curvas de entonación... & 45,1 \\
\hline Escuchar un texto y colocar signos ‘ ¡ o ¿? & 57,1 \\
\hline Adivinar el estado de ánimo de un hablante por su entonación & 46,4 \\
\hline Juegos de rol (role play), diálogos teatrales & 70,8 \\
\hline Reproducir estados de ánimo: alegría, tristeza, enfado... & 46,5 \\
\hline Memorizar un poema o un cuento & 34,0 \\
\hline Audiciones de canciones & 71,2 \\
\hline Ejercicios con anuncios de publicidad (vídeos) & 65,6 \\
\hline
\end{tabular}

Tabla 6. Tipología de las actividades de práctica prosódica. SÍ/NO

Se puede apreciar que los estudiantes han trabajado en sus países de origen con una amplia tipología de ejercicios, de los que destacan claramente aquellos que tienen que ver con el tipo 'escucha y repite', ejercicios de destrezas y listas de 
palabras; cabe mencionar, sin embargo, que no les son ajenos los ejercicios de dramatización (un 70,8\% los reconoce y afirma haberlos practicado).

Puede comprobarse, además, que el uso de canciones, de vídeos y otros elementos audiovisuales está muy asentado en la enseñanza de lenguas.

Por otro lado, y a pesar de que son estudiantes jóvenes y acostumbrados a los medios tecnológicos, pocos los utilizan para grabar su voz o utilizan el ordenador para mejorar su pronunciación.

Por último, destacan las lecturas en voz alta y los ejercicios de distinción de patrones entonativos (interrogación, exclamación, por ejemplo), pero no están acostumbrados a reproducirlo de una forma dirigida en clase.

Hay que constatar cómo los estudiantes actuales viven rodeados de tecnología, se mueven en un mundo donde lo audiovisual lo domina todo y aprenden con programas informáticos y formatos que se actualizan continuamente. No es necesario que el profesor sea un experto en todos estos avances, pero, como usuario, tiene a mano una serie de elementos que se muestran eficaces en el aula.

Los ejercicios de repetición son útiles como instrumentos de evaluación, ya que le permiten al profesor comprobar de un modo sencillo en qué medida el alumno es capaz de percibir y reproducir unos modelos, por ejemplo, una curva melódica; sin embargo, tienen un inconveniente como instrumentos de adquisición fónica, ya que consisten en que el alumno escuche y, sin pensarlo, repita. Aunque el alumno logre pronunciar correctamente en ese momento, a largo plazo no existen garantías de que será capaz de transferir esa pronunciación correcta a otra producción oral, sobre todo si se trata de una actividad de habla espontánea o en un contexto diferente al del aula.

\subsection{La fonética en los manuales de ELE}

Carbó (2003: 12) afirma que «faltan materiales que respondan a planteamientos comunicativos para el aprendizaje de la pronunciación y materiales metodológicamente adecuados para emplearlos en la corrección fonética». Con el propósito de comprobar esta falta de interés, por parte de editores y creadores de material educativo, hacia la enseñanza de la fonética en el aula de ELE, se diseñó un estudio sobre una muestra de 31 manuales disponibles en el mercado. De esos manuales solo 7 dedican una sección específica al estudio de la fonética.

En estos manuales, la representación de la fonética sobre el total de la programación puede reflejarse de la siguiente manera:

Álvarez (2000): 12-15\%; Alonso y Prieto (2011): 10\%; Borobio (1998): 8\%; Castro, Díaz y Rodero (2005): 7\%; Gaínza, Ginés, Martínez y Ordeig (2007): 10\%; Palomino (2000): 10\%; Peláez y Robles (2012): 5\%. 
En cuanto a los contenidos relacionados con la prosodia, su aparición en estos manuales es bastante limitada y suele estar relacionada con la acentuación y la pausa; solo en casos puntuales se acometen modelos de entonación. Cabe destacar el tratamiento más profundo de aspectos prosódicos, como pueden ser las curvas entonativas, los grupos fónicos, los esquemas tonales y los patrones entonativos desarrollado en la colección Sueña (Álvarez 2000). En la colección Embarque (Alonso y Prieto 2011), a partir del nivel A2 aparece de forma expresa el tratamiento de los elementos suprasegmentales.

Los manuales restantes, analizados en este artículo, no incorporan de un modo explícito el estudio de la fonética, sino que lo integran, con mayor o menor éxito, dentro de los distintos componentes de la lengua mediante un enfoque por competencias. No es extraño el que aparezcan de una forma reiterada en la introducción del autor términos como: 'comunicativo,' integral', 'enfoque por tareas', 'multidisciplinar', entre otros.

Esta integración de la fonética en las competencias ha sido habitual desde los años 80 hasta la actualidad, incluso en los manuales de tendencia claramente tradicional, como es el caso de Miquel y Sans (1983) cuya introducción afirma: «Intenta aportar modelos de trabajo originales junto a aspectos positivos de la tradición existente en la enseñanza de español para extranjeros».

Otras afirmaciones comunes en los manuales publicados en años posteriores:

Es un método comunicativo y multidisciplinar. Integra en un contexto comunicativo el aprendizaje de los diversos componentes que forman la lengua.

(Sánchez, Fernández y Díaz, 1988)

Enfoque plural e integrador, pretende reforzar las funciones comunicativas, la gramática y el vocabulario. Da importancia fundamental a la autonomía del estudiante.

(Alcoba, 2001)

«Está basado en una metodología ecléctica que se sirve de todo lo positivo de los diferentes enfoques. Todo el material que presentamos ha sido experimentado por alumnado de diferentes nacionalidades y edades».

(Moreno, Moreno y Zurita, 2004)

Concepción comunicativa de la enseñanza de lenguas basada en el enfoque por tareas. Estructura modular que facilita que cada grupo pueda establecer su propio itinerario.

(Chamorro, Lozano y Ruiz, 2006) 
Distribución modular y flexible, basándose en un enfoque orientado a la acción; las unidades se orientan a un objetivo tema que dota de contexto a las tareas propuestas.

(Menéndez, 2010)

El curso de español: en que el alumno es el centro de la enseñanza y el aprendizaje (...), que integra el manejo de internet y los soportes digitales, (...) en el que la comunicación y el aprendizaje del léxico y de la gramática se integran plenamente.

(Sans, Martín y Garmendia, 2012)

\section{Conclusiones}

Se puede confirmar, visto lo reflejado por las opiniones expresadas por los participantes en la investigación llevada a cabo, que las destrezas orales son el componente más valorado en el aula, tanto por los profesores nativos de español como por los estudiantes internacionales. Estos profesores muestran bastante coherencia entre el ideal de dedicación expresado en las encuestas y el tiempo real empleado en el aula de ELE en la realización de actividades relacionadas con las destrezas orales. No se puede decir lo mismo de los profesores en los centros de origen de los estudiantes encuestados: la enseñanza de la gramática y de las destrezas escritas gozan de la preferencia de estos profesores. En lo referente a los estudiantes internacionales de español, se percibe un claro divorcio entre lo que les gustaría estudiar y lo que realmente han estudiado en su país de origen.

Se ha podido comprobar, en lo relacionado con la importancia que se le da a la fonética en el aula de ELE, cómo entre los profesores encuestados se da la paradoja de que, a pesar de su clara preferencia hacia las destrezas orales, desdeñan el valor de la fonética y de su enseñanza en el aula (aunque esta parte de la lingüística sea un componente fundamental de la oralidad); todo ello, a pesar de que los estudiantes son conscientes de la necesidad de mejorar su pronunciación. Además, en el caso de que un profesor trabaje con la fonética lo hará generalmente con los elementos segmentales, debido, entre otras razones, a que los suprasegmentos son los componentes más complejos de una lengua y los más difíciles de ser analizados desde un plano lingüístico.

Asimismo, se da un gran desconocimiento, por parte de los profesores, de los principios teóricos sobre fonética, lo que hace que se sientan inseguros: los que han participado en las encuestas elaboradas en esta investigación consideran que, para poder tratar la pronunciación y corrección fonética en el aula, se debe 
tener, como mínimo, unos conocimientos amplios de la materia. En este sentido, hay que animar a la búsqueda de material formativo y de investigación; hoy en día, con la presencia de Internet, hay una gran cantidad de artículos (la mayoría gratuitos y de acceso libre) al alcance del profesor. Puede ser de gran utilidad, por ejemplo, como introducción a la didáctica de la pronunciación, el trabajo de Cantero (2003): en él se presentan, de forma clara y concisa, los conceptos básicos de la fonética y fonología del español y cómo transmitirlos al estudiante ${ }^{2}$.

El desconocimiento y falta de interés por la fonética, mostrado en las conclusiones de este artículo, se ve reflejado en la escasa dedicación de los manuales de ELE; en el caso de los elementos suprasegmentales es casi nula. La tipología de los materiales utilizados para la enseñanza de la fonética española sigue centrada en ejercicios de 'escucha y repite', drills, lecturas en voz alta y listados de palabras, lo que obliga a plantear la búsqueda de nuevas formas de ejercicios, menos repetitivos y más creativos. El trabajo con la pronunciación y con los elementos suprasegmentales exige ejercicios de repetición que suelen ser tediosos y que pueden dispersar al grupo; al mismo tiempo, en la práctica fonética afloran todos los miedos del estudiante a parecer ridículo. Es necesario crear una atmósfera de trabajo en la que prime la confianza entre los miembros del grupo y que sirva de estímulo para el desarrollo de los ejercicios. Este ambiente puede ser fomentado desde el uso de técnicas teatrales, como es el caso del juego dramático ${ }^{3}$, ya que mejoran la expresión oral y la competencia comunicativa, estimulan la creatividad, favorecen la interrelación entre los participantes y ayudan a superar la timidez para hablar y actuar en público.

En Boquete (2012) se muestra cómo es posible integrar el uso de técnicas teatrales a la enseñanza de la prosodia en español, al tiempo que se diseña una programación completa para el estudio de los elementos suprasegmentales en español. Esta propuesta puede consultarse en el anexo I de la tesis, está planteada para un nivel B2 y tiene como destinatario a cualquier estudiante internacional que necesite formación sobre estos elementos fonéticos.

\footnotetext{
2 Del mismo modo, en el capítulo 2.4 de la tesis doctoral Boquete (2012) se desarrolla una exposición teórica sobre los aspectos relacionados con la prosodia en español, desde la perspectiva de la Fonética Articulatoria, con el fin de aportar el conocimiento necesario para su enseñanza.

3 Se puede obtener información sobre cómo aplicar estas técnicas en la enseñanza de lenguas en Boquete (2012), concretamente en el epígrafe 2.4. de la tesis doctoral.
} 


\section{Referencias bibliográficas}

Alcón, E. (2000). «Desarrollo de la competencia oral en el aula de lenguas extranjeras: perspectivas metodológicas y de investigación». En C. MuÑoz (Ed.) Segundas lenguas. Adquisición en el aula. Barcelona: Ariel. 259-276.

Boquete, G. (2012). El uso del juego dramático en la enseñanza de lenguas: las destrezas orales. Tesis doctoral. Universidad de Alcalá. Disponible en la base Teseo: <https://www.educacion.gob.es/teseo/imprimirFicheroTesis. do?fichero $=30865>$

- (2014). «Las destrezas orales en el aula de ELE: situación actual de las competencias comunicativas». En Lenguaje y textos No 39 Revista SEDLL. Barcelona: Graó.

Canellada, M.y Kuhlmann, J. (1987). Pronunciación del español: lengua bablada y literaria. Madrid: Editorial Castalia.

Cantero, F.J. (2002). Teoría y análisis de la entonación. Barcelona: Edicions de la Universitat de Barcelona.

- (2003) «Fonética y didáctica de la pronunciación», en A. Mendoza (Coord.) Didáctica de la lengua y la literatura. Madrid: Prentice Hall. Cap.15, 545-572.

Carbó, C. (2003). «Estándar oral y enseñanza de la pronunciación del español como primera lengua y como lengua extranjera», en Estudios Lingüísticos de la Universidad de Alicante 17.161-180.

Cortés, M. (1999). Adquisición de la entonación española por parte de hablantes nativos de chino (tesis doctoral). Universidad de Barcelona: Departamento de Didáctica de la Lengua y la Literatura.

- (2001). «El papel de la prosodia en la enseñanza de la lengua extranjera: una revisión de materiales didácticos». En Lenguaje y Textos $N^{\circ} 17$. Revista SDELL.

- (2002). Didáctica de la prosodia del español: la acentuación y la entonación. Madrid: Edinumen.

GiL, J. (2007). Fonética para profesores de español: de la teoría a la práctica. Madrid: Arco Libros.

- (Ed.) (2012). Aproximación a la enseñanza de la pronunciación en el aula de español. Madrid: Edinumen.

Hidalgo, N y Cabedo, A. (2012). La enseñanza de la entonación en el aula de ELE. Madrid: Arco Libros.

Instituto Cervantes (2006). Plan curricular del Instituto Cervantes. Niveles de referencia para el español. Madrid: Instituto Cervantes-Biblioteca nueva. Quilis, A. (1999). Tratado de Fonología y Fonética españolas. Madrid: Gredos. 
Sos A, J. (1999). La entonación del español, su estructura fónica, variabilidad y dialectología. Madrid: Cátedra.

\section{Relación de manuales analizados en este artículo:}

Alcoвa, S. (Coord.) (2001). Esespañol. Madrid: Espasa Calpe.

Alonso, M. y Prieto, R. (2011). Embarque. Madrid: Edelsa.

Álvarez, M. (Dir.) (2000). Sueña. Madridः Anaya

- (Dir.) (2006). Vuela. Madrid: Anaya.

Artuñedo, B. y Johnson, C. (1995). Ele 2: curso de español para extranjeros. Madrid: SM.

Borobio, V. (1998). Ele, curso de español para extranjeros. Madrid: SM.

Castro, F; Martín, F. y Morales, R. (2004). Nuevo ven 2. Madrid: Edelsa.

Castro, F*; Díaz, P. y Rodero, I. (2005). Español en marcha. Madrid: SGEL.

Cerrolaza, M.; Cerrolaza, O. y Llovet, B. (2000). Planeta ElE. Madrid: Edelsa.

- (2008). Pasaporte. Madrid: Edelsa.

Chamorro, M.; Lozano, G. y Ruiz, G. (2006). El ventilador. Barcelona: Difusión.

Cuadrado, C.; Melero, P. y Sacristán, E* (2009). Protagonistas. Madrid: SM.

Encina, A.; Martínez, M. y Sans N. (2008). Gente Joven. Barcelona: Difusión.

Fernández, L. (2007). Aula de enlace. Barcelona: Almadraba.

Gaínza, A.; Ginés, I*; Martínez, M.y Ordeig, I. (2007). Español lengua viva. Madrid: Santillana.

García, F. (1988). Español: curso de perfeccionamiento. Universidad de Salamanca.

González, A. y Romero, C. (2004). ECO curso modular de español lengua extranjera. Madrid: Edelsa.

Marcos, M. у Obra, M. (2000). Punto final. Madrid: Edelsa.

Martín, E*; Martínez, G. y Sans, N. (1997). Gente. Barcelona: Difusión.

Menéndez, M. (2010). Etapa. Madrid: Edinumen.

- (Coord.) (2009). Prisma. Madrid: Edinumen.

Miquel, L. y Sans, N. (1983). ¿A que no sabes? Madrid: Edelsa.

Moreno, C.; Moreno, V. y Zurita, P. (2004). Avance. Madridः SGEL.

- (2010). Nuevo Avance. Madrid: SGL.

Palomino, M. (2000). Primer plano 1. Madrid: Edelsa.

Peláez, S. y Robles, A. (2012). Método de español. Madrid: Anaya.

Ruipérez, G.; Aguirre, B. y Román, E. (2002). Primer plano. Madrid: Edelsa. 
Sánchez, A.; Fernández, J. y Díaz, M.C. (1988). Antena. Madrid: SGEL.

Sánchez, J.; Moreno, C. y Santos, I. (2005). Nuevo español sin fronteras. Madrid: SGEL.

Sans, N.; Martín, E. y Garmendia, A. (2012). Bitácora. Barcelona: Difusión.

Santos, I.; Sánchez, J.; Pisonero, I. y Pinilla, R. (2005). Canal joven. Madrid: SGEL. 